\title{
INVARIANTS OF FINITE ABELIAN GROUPS ACTING ON THE ALGEBRA OF TWO $2 \times 2$ GENERIC MATRICES
}

\author{
CHAN HUH
}

(Communicated by Donald S. Passman)

\begin{abstract}
In this paper, we discuss the finite generation problem for the invariant subalgebras of finite abelian groups which act linearly on the $2 \times 2$ generic matrix algebra, and we obtain some conditions on the groups to ensure that their invariant subalgebras are finitely generated.
\end{abstract}

Introduction. Let $R_{(m)}=K\{X, Y\}$ be a generic matrix algebra generated by two $m \times m$ generic matrices over a field $K$. A theorem of Fisher and Montgomery [2] shows that if $G=\langle g\rangle$ is a finite cyclic group such that char $K+|G|$, then $R_{(m)}^{G}$, the subalgebra of invariants, is not finitely generated whenever $g$ is not scalar and $m \geq|G|-[\sqrt{|G|}]+1$.

When $m=2$ and $G$ is a finite subgroup of $\mathrm{SL}(2, K)$, Formanek and Schofield [3] have proved that if char $K+|G|$, then $R_{(2)}^{G}$ is always finitely generated.

In this paper we consider the case when $m=2$ and $G$ is a finite abelian subgroup of $\mathrm{GL}(2, K)$ such that char $K \nmid|G|$. In this case we may assume that $K$ is algebraically closed. So $G$ acts diagonally on $R=R_{(2)}$ and $R^{G}$ is generated by some monomials in the generators of $R$.

We obtain necessary and sufficient conditions on $G$ to ensure that $R^{G}$ is finitely generated. They are as follows: $R^{G}$ is finitely generated if and only if $G$ contains no pseudoreflections or, equivalently, $G=\langle g\rangle$ is cyclic and the eigenvalues of $g$ in some extension field of $K$ have the same order. In some sense, this provides a converse to Theorem 1 of Fisher and Montgomery [2].

\section{For cyclic groups.}

LEMMA 1.1 (FORMANEK AND SCHOFIELD). Let $G$ be any finite subgroup of $\mathrm{GL}(2, K)$ such that char $K \nmid|G|$ and let $[R, R]=J$ be the commutator ideal of $R$. Then

(i) for each $m \geq 1, J^{m}=J(X Y-Y X)^{m-1}$, and

(ii) $G$ acts linearly on $R / J^{2}$ and $\left(R / J^{2}\right)^{G}$ is a finitely generated $K$-algebra.

ProOF. See [3, Lemmas 6 and 7].

LEMMA 1.2. Let $u \in R$ be homogeneous and $Z=X Y-Y X$. Then

$$
u=v Z+a Y^{r} X^{s}+\sum b_{i, j, \kappa, l} Y^{i} X^{j} Z Y^{\kappa} X^{l}
$$

Received by the editors February 25, 1987 and, in revised form, August 19, 1987.

1980 Mathematics Subject Classification (1985 Revision). Primary 16A38.

Key words and phrases. Invariants of group action, generic matrix algebra.

This paper will form part of the author's doctoral dissertation at the University of Cincinnati under the supervision of Professor Joe W. Fisher. 
where $v \in R$ is homogeneous, $r, s, i, j, \kappa, l$ are nonnegative integers, and $a, b_{i, j, \kappa, l}$ are in $K$. Moreover, $u, v Z, Y^{r} X^{s}$ and $Y^{i} X^{j} Z Y^{\kappa} X^{l}$ all have the same degree in $X$ and the same degree in $Y$.

PrOOF. Since $u$ is homogeneous, $u$ is a sum of a finite number of monomials in $X$ and $Y$-all of which have the same degree in $X$ and the same degree in $Y$. Consider one of these monomials $M$. If for each $X$ in $M$ no $Y$ occurs immediately adjacent to $X$ on the right, then $M$ has the form $a Y^{r} X^{s}$. If $Y$ occurs adjacent to $X$ on the right, then replace " $X Y$ " by " $Z+Y X$ " to get $M$ with " $X Y$ " replaced by " $Y X$ " plus a new term involving $Z$. Note that this transformation preserves homogeneity. By iterating this process we reduce the original monomial $M$ to one of the form $a Y^{r} X^{s}$ at the expense of introducing some new terms of the form $N=b u_{1} u_{2} \ldots u_{t}$ where $b \in K$, one $u_{\lambda}$ is $Z$ and the rest are $X$ or $Y$.

If for each $X$ in $N$ no $Y$ occurs immediately adjacent to $X$ on the right, then $N$ has the form $b_{i, j, \kappa, l} Y^{i} X^{j} Z Y^{\kappa} X^{l}$. If $Y$ occurs adjacent to $X$ on the right, then replace " $X Y$ " by " $Z+Y X$ " to get $N$ with " $X Y$ " replaced by " $Y X$ " plus a new term involving two factors of $Z$. By iterating this process the original term $N$ is reduced to one of the form $b_{i, j, \kappa, l} Y^{i} X^{j} Z Y^{\kappa} X^{l}$ at the expense of introducing some new terms of the form $p=c u_{1}, u_{2}, \ldots, u_{t}$ where $c \in K$ and there exist $\lambda$ and $\rho$ with $1 \leq \lambda<\rho \leq t$ such that $u_{\lambda}=Z=u_{\rho}$. However, each of these new terms $P$ is in $J^{2}$. By Lemma 1.1, $J^{2}=J Z$ so $P=v Z$ for some $v \in J$. Since $P$ is homogeneous and $P=v Z$, we have that $v$ is homogeneous.

It is clear from the proof that all monomials in $u$ and $v Z, Y^{r} X^{s}, Y^{i} X^{j} Z Y^{\kappa} X^{l}$ have the same degree in $X$ and have the same degree in $Y$, i.e., $X$-deg $=s=j+l$ and $Y$-deg $=r=i+\kappa$.

In order to prove the main theorem of this section (Theorem 1.7), we need to define some integer-valued mappings. Fix integers $n$ and $t$ such that $2 \leq n$, $0 \leq t<n$, and $(n, t)=1$.

(1) for each nonnegative integer $p$, let $\alpha(p)$ be the smallest nonnegative integer such that $p(t+1)+\alpha(p) \equiv 0(\bmod n)$, i.e., $\alpha(p)=\min \{q \mid q \geq 0, p(t+1)+q \equiv 0$ $(\bmod n)\}$.

(2) For each pair of nonnegative integers $p$ and $q$, let $\beta(p, q)$ be the smallest nonnegative integer such that $p(t+1)+q+\beta(p, q) t \equiv 0(\bmod n)$, i.e.,

$$
\beta(p, q)=\min \{r \mid r \geq 0, p(t+1)+q+r t \equiv 0(\bmod n)\}
$$

(3) For each triple of nonnegative integers $p, q$, and $r$, let $\gamma(p, q, r)$ be the smallest nonnegative integer such that $p(t+1)+q+r t+\gamma(p, q, r) \equiv 0(\bmod n)$.

(4) For each quadruple of nonnegative integers $p, q, r$, and $s$, let $\delta(p, q, r, s)$ be the smallest nonnegative integer such that $p(t+1)+q+r t+s+\delta(p, q, r, s) t \equiv 0(\bmod n)$.

Since $(n, t)=1$, the values $\alpha(p), \beta(p, q), \gamma(p, q, r)$, and $\delta(p, q, r, s)$ exist uniquely for all nonnegative integers $p, q, r$, and $s$. Moreover, all these values are less than $n$, i.e., $0 \leq \alpha, \beta, \gamma, \delta<n$.

Now we define some special subsets of $R$ by using the mappings $\alpha, \beta, \gamma$, and $\delta$ defined above. 
DEFINITION.

$$
\begin{aligned}
B_{1} & =\left\{X^{\alpha(p)} Z^{p} \mid 1 \leq p \leq n\right\}, \\
B_{2} & =\left\{Y^{\beta(p, q)} X^{q} Z^{p} \mid 1 \leq p \leq n, 0 \leq q \leq n-1\right\}, \\
B_{3} & =\left\{Z Y^{\beta(p, q)} X^{q} Z^{p-1} \mid 1 \leq p \leq n, 0 \leq q \leq n-1\right\}, \\
B_{4} & =\left\{X^{\gamma(p, q, r)} Z Y^{r} X^{q} Z^{p-1} \mid 1 \leq p \leq n, 0 \leq q, r \leq n-1\right\}, \\
B_{5} & =\left\{Y^{\delta(p, q, r, s)} X^{s} Z Y^{r} X^{q} Z^{p-1} \mid 1 \leq p \leq n, 0 \leq q, r, s \leq n-1\right\}, \\
B & =B_{1} \cup B_{2} \cup B_{3} \cup B_{4} \cup B_{5} .
\end{aligned}
$$

REMARKS 1.3. (i) Since $B_{1}, B_{2}, B_{3}, B_{4}$, and $B_{5}$ are finite subsets of $R, B$ is also a finite subset of $R$.

(ii) Let $n$ be a positive integer such that char $K \nmid n$ and let $\omega$ be a primitive $n$th root of unity. If $t$ is a positive integer such that $(n, t)=1$, then we define an automorphism $g$ on $R$ by setting $X^{g}=\omega X$ and $Y^{g}=\omega^{t} Y$. By the definition of the mappings $\alpha, \beta, \gamma$, and $\delta$, we have that $B$ is a subset of $R^{G}$ where $G=\langle g\rangle$.

(iii) If $G$ is as in (ii) and $0 \neq u \in R^{G}$ is homogeneous, then each monomial in $u$ lies in $R^{G}$.

LEMMA 1.4. Let $G=\langle g\rangle$ be a cyclic group of order $n$ acting on $R$ by $X^{g}=\omega X$ and $Y^{g}=\omega^{t} Y$ where $\omega$ is a primitive $n^{\text {th }}$ root of unity such that char $K \nmid n$ and $(t, n)=1$. Let $p$ be a positive integer.

(i) If $Y^{i} X^{j} Z^{p} \in R^{G}$ for some nonnegative integers $i$ and $j$, then there exist $w$ in $R^{G}$ and $b$ in $B$ such that $Y^{i} X^{j} Z^{p}=w b$.

(ii) If $Y^{i} X^{j} Z Y^{\kappa} X^{l} Z^{p} \in R^{G}$ for some nonnegative integers $i, j, \kappa, l$ then there exist $w$ in $R^{G}$ and $b$ in $B$ such that $Y^{i} X^{j} Z Y^{\kappa} X^{l} Z^{p}=w b$.

PROOF. The proof of (i) is similar to that of (ii), so we will prove (ii). If $p \geq n$, then $Y^{i} X^{j} Z Y^{\kappa} X^{l} Z^{p}=\left(Y^{i} X^{j} Z Y^{\kappa} X^{l} Z^{p-n}\right) Z^{n}$. Let $b=Z^{n}$ and $w=$ $Y^{i} X^{j} Z Y^{\kappa} X^{l} Z^{p-n}$. Then $b \in B_{1} \subset B \subset R^{G}$. Since $R$ has no zero divisors, $w$ must be in $R^{G}$.

Now assume that $p<n$. For convenience let $u=Y^{i} X^{j} Z Y^{\kappa} X^{l} Z^{p}$.

(1) If $l \geq \alpha(p)=\alpha$, then let $b=X^{\alpha} Z^{p}$ and $w=Y^{i} X^{j} Z Y^{\kappa} X^{l-\alpha}$. Then $u=w b$ and $b \in B_{1} \subset B$. Again $w \in R^{G}$.

(2) If $l<\alpha(p)$ and $\kappa \geq \beta(p, l)=\beta$, then let $b=Y^{\beta} X^{l} Z^{p}$ and $w=Y^{i} X^{j} Z Y^{\kappa-\beta}$. Then $b \in B_{2} \subset B, u=w b$, and $w \in R^{G}$.

(3) If $l<\alpha(p), \kappa<\beta(p, l)$, and $\kappa=\beta(p+1, l)$, then let $b=Z Y^{\kappa} X^{l} Z^{p}=$ $Z Y^{\beta(p+1, l)} X^{l} Z^{p}$ and $w=Y^{i} X^{j}$. Then $b \in B_{3} \subset B, u=w b$ and $w \in R^{G}$.

(4) If $l<\alpha(p), \kappa<\alpha(p, l), \kappa \neq \beta(p+1, l)$, and $j \geq \gamma(p+1, l, \kappa)=\gamma$ then let $b=X^{\gamma} Z Y^{\kappa} X^{l} Z^{p}, w=Y^{i} X^{j-\gamma}$. Then $b \in B_{4} \subset B, u=w b$ and $w \in R^{G}$.

(5) If $l<\alpha(p), \kappa<\beta(p, l), \kappa \neq \beta(p+1, l), j<\gamma(p+1, l, \kappa)$, then $i \geq \delta=$ $\delta(p+1, l, \kappa, j)$. For otherwise $u \notin R^{G}$. So let $w=Y^{i-\delta}$ and $b=Y^{\delta} X^{j} Z Y^{\kappa} X^{l} Z^{p}$. Then $u=w b, b \in B_{5} \subset B$ and $w \in R^{G}$. The lemma is proved.

PROPOSITION 1.5. Let $G=\langle g\rangle$ be a cyclic group of order $n$ acting on $R$ by $X^{g}=\omega X$ and $Y^{g}=\omega^{t} Y$ where $\omega$ is a primitive $n^{\text {th }}$ root of unity such that char $K \nmid n$ and $(t, n)=1$. If $u \in R$ is homogeneous and $u Z^{p} \in R^{G}(p \geq 1)$, then $u Z^{p}=\sum w_{\nu} b_{\nu}$ is a finite sum where $w_{\nu} \in R^{G}$ and $b_{\nu} \in B$. Moreover $u Z^{p}$ and $w_{\nu} b_{\nu}$ have the same degree in $X$ and the same degree in $Y$. 
PROOF. The proof is by reverse induction on $p$. If $p \geq n$ then $Z^{n} \in B_{1} \subset B$ and $u Z^{p}=\left(u Z^{p-n}\right) Z^{n}$. Let $w=u Z^{p-n}$ and $b=Z^{n}$. Then $u Z^{p}=w b$ and $w \in R^{G}$. Assume $1 \leq p<n$. By Lemma 1.2 we have that

$$
u Z^{p}=v Z^{p+1}+a Y^{r} X^{s} Z^{p}+\sum b_{i, j, \kappa, l} Y^{i} X^{j} Z Y^{\kappa} X^{l} Z^{p}
$$

where $v \in R$ is homogeneous, $a, b_{i, j, \kappa, l} \in K$, and $u Z^{p}, v Z^{p+1}, Y^{r} X^{s} Z^{p}$ and $Y^{i} X^{j} Z Y^{\kappa} X^{l} Z^{p}$ all have the same degree in $X$ and the same degree in $Y$. By Remark 1.3(iii), each term on the right-hand side of (*) lies in $R^{G}$. From the homogeneity of $v$ and the reverse induction hypothesis $v Z^{p+1}=\sum w_{\nu} B_{\nu}$ for some $w_{\nu} \in R^{G}$ and $b_{\nu} \in B$. The other terms on the right hand side of $(*)$ are also of the form $w b, w \in R^{G}, b \in B$ by Lemma 1.4. Now the proof is completed.

THEOREM 1.6 [2, THEOREM 1]. Let $G=\langle g\rangle$ be a cyclic group acting on $R$ by $X^{g}=\omega X$ and $Y^{g}=\lambda Y$ where $\omega$ and $\lambda$ are nth roots of unity. If $\omega$ and $\lambda$ have distinct orders, then $R^{G}$ is not finitely generated.

We prove the converse of the above theorem. The proof is similar to that of $[3$, Theorem 8].

THEOREM 1.7. Let $G=\langle g\rangle$ be a cyclic group of order $n$ acting on $R$ by $X^{g}=$ $\omega X$ and $Y^{g}=\lambda Y$ where both $\omega$ and $\lambda$ have the same (multiplicative) order $n$. Then $R^{G}$ is finitely generated.

ProOF. We may assume that $\lambda=\omega^{t}$ where $(n, t)=1$. Since char $K \nmid|G|$, the natural homomorphism $\theta: R^{G} \rightarrow\left(R / J^{2}\right)^{G}$ is surjective. By Lemma 1.1(ii) there exist $v_{1}, v_{2}, \ldots, v_{m}$ in $R^{G}$ whose images generate $\left(R / J^{2}\right)^{G}$ as a $K$-algebra. As we stated before, we may assume that $v_{1}, v_{2}, \ldots, v_{m}$ are monomials since $G$ consists of diagonal matrices. Let $A=\left\{v_{1}, v_{2}, \ldots, v_{m}\right\}$ and let $T$ be the subalgebra of $R^{G}$ which is generated by $A \cup B$ where $B$ is as in the definition above. Then $T$ is finitely generated and it suffices to prove that $R^{G}=T$. It is obvious that $T \subset R^{G}$. Let $u$ be any homogeneous element of $R^{G}$. We will show that $u \in T$ by induction on the degree of $u$. If $\operatorname{deg} u=0$, then $u \in K \subset T$. Assume that $\operatorname{deg} u \geq 1$. Since $\bar{A}=\left\{\bar{v}_{1}, \bar{v}_{2}, \ldots, \bar{v}_{m}\right\}$, where $\bar{v}$ denotes the image of $v \in R^{G}$ under $\theta$, generates $\left(R / J^{2}\right)^{G}$, there exists a polynomial $f=f\left(x_{1}, x_{2}, \ldots, x_{m}\right)$ in the free algebra $K\left\langle x_{1}, x_{2}, \ldots, x_{m}\right\rangle$ such that $\bar{u}=f\left(\bar{v}_{1}, \bar{v}_{2}, \ldots, \bar{v}_{m}\right)$. So $u-f\left(v_{1}, v_{2}, \ldots, v_{m}\right) \in$ $\operatorname{Ker} \theta=R^{G} \cap J^{2} \subset J^{2}$. By Lemma 1.1(i), $u-f\left(v_{1}, v_{2}, \ldots, v_{m}\right)=v Z$ for some $v \in J$. Moreover $v Z=u-f\left(v_{1}, v_{2}, \ldots, v_{m}\right)$ is in $R^{G}$. Since each $v_{i}$ is a monomial and $J^{2}$ is homogeneous, we may assume that $v$ is homogeneous and $\operatorname{deg} v Z \leq \operatorname{deg} u$. So by Proposition 1.5, vZ $=\sum w_{\nu} b_{\nu}$ where $w_{\nu} \in R^{G}$ and $b_{\nu} \in B$. But $\operatorname{deg} w_{\nu}<\operatorname{deg} w_{\nu}+\operatorname{deg} b_{\nu}=\operatorname{deg} w_{\nu} b_{\nu}=\operatorname{deg} v Z \leq \operatorname{deg} u$ for each $\nu$, so by the induction hypothesis, $w_{\nu} \in T$. Hence $v Z=\sum w_{\nu} b_{\nu} \in T$. Thus $u=v Z+f\left(v_{1}, v_{2}, \ldots, v_{m}\right) \in T$ and so $R^{G}=T$ is finitely generated.

DEFINITION A diagonalizable $m \times m$ matrix $g$ over $K$ is said to be a pseudoreflection if $\operatorname{rank}\left(g-I_{m}\right)=1$, where $I_{m}$ is the $m \times m$ identity matrix or, equivalently, if 1 is an eigenvalue of $g$ of multiplicity exactly $m-1$.

If $g \in \mathrm{GL}(2, K)$ has eigenvalues $\omega_{1}$ and $\omega_{2}$ which are roots of unity of distinct orders, then for some $n \geq 1, g^{n}$ is a pseudoreflection. Hence for a finite subgroup $G$ of $\mathrm{GL}(2, K)$ such that char $K+|G|$, the following two statements are equivalent: (i) $G$ has an element $g$ whose eigenvalues have distinct orders, and (ii) $G$ contains a pseudoreflection. 
COROLLARY 1.8. Let $G$ be a cyclic subgroup of $\mathrm{GL}(2, K)$ of order $n$ with char $K \nmid n$. Then $R^{G}$ is finitely generated if and only if $G$ contains no pseudoreflections.

ProOF. Let $g$ be the generator of $G$. Since we may assume that $K$ is algebraically closed, $g$ is diagonalizable.

If $R^{G}$ is finitely generated, then the eigenvalues of $g$ have the same order by Theorem 1.6. So $G=\langle g\rangle$ cannot contain pseudoreflections.

Conversely, if $G$ contains no pseudoreflections, then the eigenvalues of $g$ must have the same order. So $R^{G}$ is finitely generated by Theorem 1.7.

2. For abelian groups. In this section, we let $G$ be a finite abelian subgroup of $\operatorname{GL}(2, K)$ such that $2 \leq|G|$ and char $K+|G|$. Since we may assume that $K$ is algebraically closed, $G$ can be considered as a group of diagonal matrices.

First we need the following result of Fisher and Montgomery [2, Proposition 1].

PROPOSITION 2.1. Let $n$ and $N$ be positive integers and let $f(x, y)$ be a nonzero homogeneous polynomial in $K\langle x, y\rangle$ of $x$-degree $n+1$ and $y$-degree $N$ of the form $f(x, y)=x^{n} y^{N} x+y f_{1}(x, y)+f_{2}(x, y) y$ for some $f_{i} \in K\langle x, y\rangle$. Then $f(x, y)$ is not an identity for $m \times m$ matrices for any $m \geq 2$.

LEMMA 2.2. Let $G$ be a finite abelian subgroup of $\mathrm{GL}(2, K)$ such that char $K \nmid$ $|G|$. If $R^{G}$ is finitely generated then $X Y^{q} \in R^{G}$ and $X^{p} Y \in R^{G}$ for some $p \geq 1$ and $q \geq 1$.

ProOF. Suppose $R^{G}$ is finitely generated. By symmetry it suffices to prove $X Y^{q} \in R^{G}$ for some $q \geq 1$. Let $m$ be the smallest positive integer such that $X^{m} Y^{q} \in R^{G}$ for some $q \geq 1$; such an $m$ exists since $X^{n} Y^{n} \in R^{G}$ where $|G|=n$. Hence $X^{m} Y^{q+n \kappa} \in R^{G}$ for each $\kappa \geq 0$. Assume $m \geq 2$. Note if $u \in R^{G}$ and $v \in R$ are monomials such that $u$ and $v$ have the same degree in $X$ and the same degree in $Y$, then $v \in R^{G}$ since $G$ is diagonalizable. Hence $X^{m-1} Y^{q+\kappa n} X \in R^{G}$ for each $\kappa \geq 0$. Since $m-1 \geq 1, X^{m-1} Y^{q+\kappa n} X$ has no proper initial segments which lie in $R^{G}$ by definition of $m$. Also note that since $G$ is diagonal, if $u \in$ $R^{G}$ is homogeneous, then each monomial of $u$ lies in $R^{G}$. So we may choose a set of generators $\left\{u_{1}, u_{2}, \ldots, u_{r}\right\}$ of $R^{G}$, all of which are monomials in $X$ and $Y$. Let $\kappa$ be an integer greater than the maximal of the degrees of $u_{i}$ 's. Since $X^{m-1} Y^{q+\kappa n} X \in R^{G}$, it is a finite sum of monomials in $u_{1}, u_{2}, \ldots, u_{r}$, that is $X^{m-1} Y^{q+n} X=\sum a_{i} u_{i_{1}} u_{i_{2}}, \ldots, u_{i_{s}}$ where $i=\left(i_{1}, i_{2}, \ldots, i_{s}\right)$ and $a_{i} \in K$. Since $R^{G}$ is homogeneous, we may assume that each $a_{i} u_{i_{1}} u_{i_{2}}, \ldots, u_{i_{s}}$ has $X$-degree $m$ and $Y$ degree $q+\kappa n$. Consider any monomial $a_{i} u_{i_{1}} u_{i_{2}}, \ldots, u_{i_{s}}$ appearing in this expression of $X^{m-1} Y^{q+\kappa n} X$. Then $s>n \geq 2$, since $\kappa n<m+q+\kappa n=\operatorname{deg}\left(X^{m-1} Y^{q+\kappa n} X\right)=$ $\operatorname{deg}\left(a_{i} u_{i_{1}} u_{i_{2}}, \ldots, u_{i_{s}}\right)<\kappa s$. Since the $X$-degree of $a_{i} u_{i_{1}} u_{i_{2}}, \ldots, u_{i_{s}}$ is $m$ and $u_{i_{j}} \in$ $R^{G}$ for each $j=1,2, \ldots, s$, from the definition of $m$, exactly one $u_{i_{j}}$ has $X$ as its factor. Thus each monomial $a_{i} u_{i_{1}} u_{i_{2}}, \ldots, u_{i_{s}}$ has the form either $v Y$ or $Y v$ for some $v$ in $R$. But this is impossible by Proposition 2.1. Hence $m=1$.

Actually the converse of Lemma 2.2 is true.

THEOREM 2.3. Let $G$ be a finite abelian subgroup of $\mathrm{GL}(2, K)$ such that char $K$ $\nmid|G|$. Then the following are equivalent:

(i) $R^{G}$ is finitely generated, 
(ii) $G$ contains no pseudoreflections,

(iii) $G=\langle g\rangle$ is cyclic and the eigenvalues of $g$ in some extension field of $K$ have the same order.

Proof. (iii) implies (i) is just Theorem 1.7.

For (i) implies (ii), assume that $G$ contains a pseudoreflection $g$. By symmetry we may assume $g=\left(\begin{array}{ll}\omega & 0 \\ 0 & 1\end{array}\right)$, for some $\omega \neq 1$. Then for any $q \geq 1,\left(X Y^{q}\right)^{g}=$ $\omega X Y^{q} \neq X Y^{q}$. Since $X Y^{q} \notin R^{G}$ for any $q \geq 1, R^{G}$ is not finitely generated by Lemma 2.2.

For (ii) implies (iii), suppose that $G$ contains no pseudoreflections. If $G$ is not cyclic, then $G=G_{1} \oplus \cdots \oplus G_{r}$ where each $G_{i}=\left\langle g_{i}\right\rangle$ is cyclic of order $n_{i}$ with $n_{1}\left|n_{2}\right|, \ldots, \mid n_{r}$ and $r \geq 2$. Hence for some primitive $n_{r}^{\text {th }}$ root of unity $\omega$, the generators $g_{1}$ and $g_{r}$ have the following forms:

$$
g_{1}=\left(\begin{array}{cc}
\omega^{a} & 0 \\
0 & \omega^{b}
\end{array}\right) \text { and } g_{r}=\left(\begin{array}{cc}
\omega & 0 \\
0 & \omega^{t}
\end{array}\right)
$$

where $a t \not \equiv b\left(\bmod n_{r}\right)$ since $g_{1} \notin\left\langle g_{r}\right\rangle$. But

$$
g_{1} g_{r}^{-a}=\left(\begin{array}{cc}
1 & 0 \\
0 & \omega^{b-a t}
\end{array}\right)
$$

is a pseudoreflection since $\omega^{b-a t} \neq 1$. This gives us the desired contradiction. So $G$ is cyclic. Since $G$ contains no pseudoreflections, the eigenvalues of the generator $g$ of $G$ have the same order.

COROLLARY 2.4. Let $G$ be as in the theorem and suppose that $|G|$ is not a prime number. Then $R^{G}$ is finitely generated if and only if $R^{H}$ is finitely generated for each proper subgroup $H$ of $G$.

PROOF. If $R^{G}$ is finitely generated, then by the theorem, $G$ (and each subgroup $H$ ) contains no pseudoreflections. Thus using the theorem again, $R^{H}$ is finitely generated.

Conversely if $R^{G}$ is not finitely generated, then $G$ contains a pseudoreflection $g$. We may assume that $g$ has prime order; if not, replace $g$ by some power $g^{t}$. But then $H=\langle g\rangle$ has prime order, and so $H \neq G$ since $|G|$ is not prime. Thus $R^{H}$ is not finitely generated, for some proper subgroup $H$ of $G$.

The following corollary was noticed by R. Guralnick.

COROLLARY 2.5. Let $H$ be a finite subgroup of $\mathrm{GL}(2, K)$ of odd order such that char $K \nmid|H|$. Then $R^{H}$ is finitely generated if and only if $H$ contains no pseudoreflections.

PROOF. As we stated before, we may assume that $K$ is algebraically closed. So, if $n$ is the degree of an irreducible representation, then $n$ must divide $|H|$ by $[\mathbf{1}$, Theorem 53.17]. By Theorem 2.3, it is enough to show that $H$ is abelian. Let $V$ be a 2-dimensional vector space over $K$. Then $H$ is a group of linear operators of $V$. If $n$ is the degree of an irreducible representation of $H$, then $n$ is odd since $n|| H \mid$. Hence the $K$-dimension of any irreducible $K[H]$-submodule of $V$ is odd and equal to or less than $2=\operatorname{dim} V$, so it is 1 . Thus $H$ is diagonalizable, so $H$ is abelian.

Note. A natural question is whether our theorem can be extended to finite solvable groups. An obstacle to extending it to finite solvable groups-by the 
obvious approach-is a theorem of R. M. Guralnick [4] which states that $R^{G}$ cannot be isomorphic to a generic matrix algebra.

ACKNOWLEDGMENT. The author wishes to express his deepest appreciation to Professor Joe W. Fisher for his helpful advice and encouragement during preparation of this paper. Also the author is grateful to the referee for thoughtful comments which have greatly improved the exposition.

\section{REFERENCES}

1. C. W. Curtis and I. Reiner, Representation theory of finite groups and associative algebras, Wiley, New York, 1962.

2. J. W. Fisher and S. Montgomery, Invariants of finite cyclic groups acting on generic matrices, J. Algebra 99 (1986), 430-437.

3. E. Formanek and A. H. Schofield, Groups acting on the ring of two $2 \times 2$ generic matrices and a coproduct decomposition of its trace ring, Proc. Amer. Math. Soc. 95 (1985), 179-183.

4. R. M. Guralnick, Invariants of finite groups on relatively free algebras, Linear Algebra Appl. 72 (1985), 85-92.

Department of Mathematics, Pusan National University, Pusan 609-735, KoREA 